Volume 8, No.4, July - August 2019

International Journal of Advanced Trends in Computer Science and Engineering

Available Online at http://www.warse.org/IJATCSE/static/pdf/file/ijatcse93842019.pdf

https://doi.org/10.30534/ijatcse/2019/93842019

\title{
Innovative Drug and Disease Prediction with Dimensionality Reduction and Intelligence Based Random Walk Methods
}

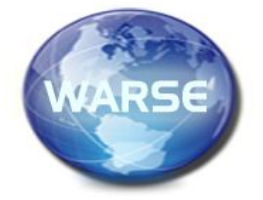

\section{ABSTRACT}

Medicine is a chemical, which processes, cures, avoids or diagnoses illnesses. There are three phases in drug design: phase of discovery, preclinical phase, and phase of clinical development [1]. Associations of drug-disease reveal and have received excellent attention to the close relationship between drugs and illnesses. Computing techniques may monitor prospective sedate-illness Linkages and additional or expensive moist experiments are performed.

A variety of computational methods for predicting sedate disease associations have been suggested in the latest years. Many medicine-sickness confederacies have been identified and saved in databases. Many organizations are however unaccounted for and need to be found. However, there have been few algorithms dedicated to medicines for a distinct illness. This is also one reason why many findings are not sufficiently predictive. The most prevalent form of hepatocellular carcinoma (HCC) in drug discovery. The second most common hepatobiliary disease following HCC is the Cholangiocarcinoma (CCA), representing 10 to 15 half of all liver carcinomas.

Key words: Hepatocellular carcinoma (HCC), Drug-Disease associations, Drug-Disease Discovery, Dimensionality Reduction, Computational Intelligence, Random Walk Methods.

\section{INTRODUCTION}

The second most common cancer and the fifth leading cause of human death are hepatitis cell (HCC) and lung cancer [2]. $\mathrm{HCC}$, recognized as a Hepatocellular malignant disease, is a significant health issue with an annual amount of more than 700,000 deaths in the world. It mainly influences liver cirrhosis patients and is presently the most prevalent cause of death. However, the therapeutic choices for HCC patients are limited and effective [3].

In this study, the following techniques are implemented, suggested, to forecast more and more accurate HCC medication signs. Predicting Novel drug disorders for HCC related on Multi-source simulated Random Walk (PDD-MSSARW), Multi-Source Imperialist Competitive Algorithm derived Random Walk (PDD-MBARW), Multi-Source Cuckoo Search Random Walk (PDD-MCSRWA) which comprises of several key measures. For one thing, a GWIN network centered on gene grimace and the jungle for protein interaction (PIN) is constructed. The decrease in dimensionality has done with the reduction techniques. Build a network of drug-drug similarities hinges on multi-source random walking techniques implemented in GWIN. Finally, all drugs have selected in a pharmaceutical network for resemblance, depending on HCC and CCA recognized drugs.

\section{LITERATURE REVIEW}

Gottlieb and other researchers [4] offered a fresh method to handle approved drugs and new molecules, the massive prediction of drug indications (PREDICT). Confirm PREDICT probability of overlap with pharmaceutical indications currently undergoing medical testing and their correspondence to tissue-based expression information on the drug goals.

Li et al[ 5] introduced a technology that includes mining and text mining techniques of molecular interactions. This specific genetic seed is increased and rearranged through its reprioritization in molecular interaction networks related to diseases in the functional context.

The variations in the processes in a single gene, Yu et al[6] have provided a new technique for defining both therapeutic and unpleasant HCC medicines based on tissue-specific pathways taking both hepatological tissue and functional pathways. Three drugs and three aggravating drugs for HCC have finally gathered by filtering the Comparative Toxicogenomics Database (CTD) criterion.

Luo and his team.[ 7] launched a fresh software pipeline recognized as the Drug-Target Interaction Net to acknowledge fresh pharmaceutical reactions from its built-in heterogeneous network. These findings show that DTINet can offer a practical means of integrating heterogeneous data to recognize fresh and up-to-date medications. 


\section{PROBLEM SPECIFICATION}

Repositioning of drugs is one of the key approaches to improve the method of drug detection. Most prior medicaments have not focused on a particular disease and often used to predict medication-disease combinations from a particular source of information. It is a major and urgent challenge to develop computational techniques for preventing uncontrolled medications associations, but there are still a number of problems.

- Gene-gene network formation

- Dimensionality Reduction

- drug-drug similarity network

- Diseases Prediction

To overcome the above issues various methods for dimensionality reduction and disease predictions have implemented. The accuracy of the results has improved by applying various reduction and optimization methods.

\section{PROPOSED RESEARCH CONTRIBUTION AND METHODOLOGY}

The following methodologies for achieving the goals introduced in the suggested job. Each technique performs according to measures such as the width, accuracy, precision, retrieval, and f-message of the silhouette. The suggested research utilizes the following gene network development, dimensional reduction, drug similarity networks, and disease prediction techniques.

\section{Method 1}

Predictions of new drugs and illness multifunctional

Hepatocellular carcinoma

Method 2

Improved Decomposition of Singular Value for Predictions of Hepatocellular HCC Based on Multiple-Source Bat Algorithm Random Walk

Method 3

Enhanced Random Walk for Drug and Disease Pre diction Improved Regularized Linear Discriminant Analysis (IRLDA) of HCC and ICA related on a Random Random W alk for Prediction

\section{Method 4}

The following chapter contains all these methods for a Fisher Linear Discriminant Analysis of Drugs Prediction and Diseases based on the Multi-Source Search of Cuckoo Random Walk Algorithm.

\subsection{Predictions for New Medications and Diseases Based on Multi-Source, Simulated Random Walk}

This study provides a multi-sources simulated random walk (PDD-MSSARW) integrated framework to prognosticate new medications as well as HCC maladies. Based mainly on gene expression and a network of protein interactions, the Gene-Gene Interaction Network (GWIN) is established.
Then, create a drug similarity network, depending on random walk multi-source in GWIN, with the aid of the Weighted Bipartite Graphs Network Similarity (SWBGN), which boosts up the drug buds and linking one node to another that explains the diagnosis. The strengths and overlaps of the likelihoods, as well as types of literature shown to have the effect of monitoring with those indicated on the Comparative Toxicogenomics Database (CTD), creative medication indications are efficient.

This section describes prevalent genes in the Protein-Protein Interaction network (PPI) related to their related border. The Entrez gene ID for each gene attached to the ' I ' and ' J ' pairs where the Pearson Correlation Coefficient (PCC) is associated between them and it can calculated for its $\mathrm{p}$-value. In contrast, with a PCC value of as p 0.05 , the border will be removed between the two genes' I' and' j.' Similarly, a weighted GWIN network of 11.723 genes and 80.308 edges is available.

Network for drug-drug resemblance: Get a set of genetic objectives for each drug. The UniProt ID has mapped onto the respective Entrez gene ID of each target medicine. The connection between all medicines must known to establish a network for the resemblance between medicines. By evaluating, the interrelation amid their target sets, the connection of two sedates has obtained. The strength of the relationship between two target sets must be measured by reducing the researcher's random walkers ' process[8 ]with simulated annual randomization[9] (MSSARW) on GWIN.SA is performed according to the physical method of the reinforcement of the fabric to enable the electrons to move freely in a warm tub. The gene is then heated and refreshed sensibly and leisurely in order to ensure that the drugs can become a good combination in the entire drug or low-energy condition.

Disease Drug Similarity Network: Sedate -associated genes that link to solitary another recommend that they are prone to communicate with me in an identical area and to generate a module of a disease or drug, a linked subgraph, which includes all molecular techniques of disease or drug. Disease-Drug Similarity Network: There is thus an important step in determining strong drug-disease interactions in the accurate evaluation of associations between disease modules and drug modules.

Affinity Weighted Bipartite Graph Network (SWBGN): A graph of the $\mathrm{X}$ and $\mathrm{Y}$ genes in which the borders of one $\mathrm{X}$ node to another of the $\mathrm{Y}$ node have been left is a two-party graph[10 ]. Take each edge in the bipartisan graph with one weight $\mathrm{w}$, achieve a weighted bipartisan graph. In using a bipartite graph, only a single set of genes (X or Y), when connected to the comparable node in the initial graph, looks like corners from one node to another. The dimensionality of 
genes can still decreased so that significant genes can only go beyond the classification method to enhance the precision of the outcomes obtained. In order to address the problem of gene selection and enhance the predictability, study work on Singular Value Decomposition (SVD) proceeded.

\subsection{Enhanced Decomposition of Singular Value for Prediction Of Hepatocellular Carcinoma Medications And Diseases Based On Multiple-Source Bat Random Algorithm}

A feature choice is implemented before continuing with the sedate - medication similarity network method to optimize the precision of the outcomes acquired in prior work. For a decrease of dimensionality, a novel improved algorithm for the single value decomposition (SVD) has provided. This study provides an integrative structure for $\mathrm{HCC}$ related to the Multi-Source Bat Random Walk based Algorithm (PDD-MBARW) to recognize new drugs and diseases. Build a network for the resemblance of drugs to disease drugs, a network for the resemblance of disease drugs, according to GWIN random multi-source walking.

Finally, the two-part disease medicine network scored every single medicament in the pharmaceutical resemblance web depending on the known HCC medicines. The decrease in dimensionality can only achieved by dropping columns, e.g. those that can be identified with others as collinear or acknowledged as not primarily predictive about the goal as identified by a rating attribute. On the other side, new columns that depend on linear combinations of the initial columns can also obtain. In this SVD method, gene information has considered to the $\mathrm{m}$ to $\mathrm{n}$ matrix. Decreased gene information has only used for the pharmaceutical similarity process network.

GWIN introduces various random-walkers [11] to build a drug similarity network with the bat algorithm (BA)[12] known as MSBARW.

The main feature, therefore, is the conduct of echolocation. The bats are genes that are similar to the medication used for the disease. They are also genes. When selecting a solution from the best accessible options, a random walking path has used to create a fresh local alternative for each bat. Get Sij by allocating probabilities in comparison to gene characteristics in the target set with whole TA and 0 gene neighbors.

\subsection{Improving Random Walk to Drug and Disease Prediction Improved Regulatory Linear Discriminant Analyzes (IRLDA) with Multi-Source Imperialist Competitive Algorithm based Random Walk (MICARW)}

To increase its precision, the suggested technique identifies new HCC drugs and illnesses using predetermined novel gene exposition information, gene set, and the pharmacy-related coincidence chain to develop a GWIN for HCC hinge on multisource Imperialist Competitive Random Walk (PDDH-MICARW). With the help of improved regularized linear discrimination analyzes (IRLDA), information dimensionality is reduced to strengthen the precision of forecasting. After this, a network for similarities between illnesses and medications and medicines has established, based on GWIN. Additionally, use Z score and cutoff for the evaluation and filtering of the sedate chain in the illness/sedate resemblance network. Finally yet importantly, all the drugs in the networks are chosenoidally used in the shape of drug crops to identify new potential HCC medicine.

All the original population colonies have split among the imperialist countries in the MICARW algorithm, based on their GWIN weight matrices [13]. The colonists start the motion toward their imperialist nations after separating all the territories among the imperialists. The complete GWIN weight matrix of all empires relies on the imperialist countries ' GWIN weight matrix and their colonies ' GWIN weight matrix. Remove any empire that cannot remain in the rivalry and does not boost the GWIN weight matrix shall, when imperialistic rivalry starts, from the imperialistic competition. The algorithm proceeds with the above steps until a stop has met. The results of the experiments show that the technique proposed achieves higher precision, predictability, and reminder and F-measurement results in conjunction with current methods. Cholangiocarcinoma (CCA) is a heterogeneous bile duct differentiating group of malignancies.

\subsection{Fisher Linear Discriminant Drug and Cholangiocarcinoma Prediction Analysis Relying on Multi-Source Cuckoo Search Random Walk Algorithm}

A new integrative CCA drug-driven framework with a multi-source Cuckoo Search random walk algorithm (MCSRWA) could predict early liver tumors preoperatively. First, build a GWIN based on gene expression and the network of protein interactions. The Fisher Linear Discriminant Analysis (FLDA) has used for dimension reduction. Build a similarity drug network based on MCSRWA in GWIN. Finally, all medicines in the network for substance resemblance have based on known CCA medicines. The MCSRWA also-related impacts of drug combinations on the CCA have specifically suggested.

In relation to dimensional decrease, FLDA has used for binary classification tasks. In FLDA, the information estimated to "W," which maximizes the separation measurement. The conventional LDA has designed to find the perfect linear transformation that preserves the class structure, both in the original and in a small dimension space. Build the network of similarities between medicines to make all drugs aware of the connection. In calculating the 
correlation between its goals, set the connection of two medicines can extracted. The strength of the connection between the two target groups shall measured by imitating the MCSRWA method at GWIN. Therefore, exploration and exploitation conduct may slow down the process of searching for similarities between medicines. Therefore, it is possible to slow down the search process of resemblance between drugs by involving a set step in exploration and exploitation conduct.

\section{RESULTS AND DISCUSSION}

The present PDD-MICARW, PDD-MSBARW and HCC drug predictions has evaluated in this chapter with comparative performance results to prior HCC-MBARW, PDD-MSSARW, and PDD-MRW and PREDICT approaches. In this chapter, the results has evaluated and compared. Obtain the human HCC gene set from CTD (2014version) [14], mainly. After that, the Gene Expression Omnibus (GEO)http:/www.ncbi.nlm.nih.gov/geo/) data sets (GDS) digitize HCC gene interpretation portraits [15]. The Drug Bank database incorporates all data on drugs [16], and the Drug Bank (version 4.0) [17] through drug feedback.[ 16]. Include five sets with five distinct cutoff numbers $\{1.25,2.5$, $5,10$ and 15$\}$.

Figure 1 explains some of the drug names from the outcome.
1. $(2 \mathrm{E}, 4 \mathrm{E}, 6 \mathrm{E}, 10 \mathrm{E})-3,7,11,15$-tetramethyl-
2,4,6,10,14-hexadecapentaenoic acid,

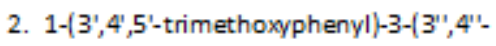
dimethoxy-2'-hydroxyphenyl)propane
3. 1-hexylcarbamoyl-5-fluorouracil
4. $2-(\leftrightarrow(2-$
chloroethyl)nitrosoamino)(carbonyl)a
mino)propanamide
5. 2-(2-hydroxyethylsulfanyl)-3-methyl-
1,4-naphthoquinone
6. 2-amino-8-(4-(2-
hydroxyethoxy)cyclohexyl)-6-(6-
methoxypyridin-3-yl)-4
methylpyrido(2,3-d)pyrimidin-7 $(8 \mathrm{H})$ -
one
7. 2'-benzoyloxycinnamaldehyde

Figure 1: Medicines discovered by our Research.

\section{An assessment of performance}

The accuracy is determined as the rate of favorable findings precisely determined for all expected beneficial findings.
Precision $=\mathrm{TP} / \mathrm{TP}+\mathrm{FP}(1)$

Sensitivity described the proportion of the favorable observation properly recognized to real-class general findings - yes.

$$
\text { Recall }=\mathrm{TP} / \mathrm{TP}+\mathrm{FN} \quad(2)
$$

F1 score has described by both precision and recall as the weighted average. Consequently, fake positives and fake negatives are required.

F1 Score $=2 *($ Recall $*$ Precision $) /($ Recall + Precision $)$

The accuracy in positive and negative conditions is determined as follows:

$$
\text { Accuracy }=(\mathrm{TP}+\mathrm{FP}) /(\mathrm{TP}+\mathrm{TN}+\mathrm{FP}+\mathrm{FN})
$$

Table 1 represents the performance comparison results of the six methods with different cutoff ranges.

Table:1. Drug Bank compared metrics of more than 20

\begin{tabular}{|c|c|c|c|c|c|c|c|}
\hline $\begin{array}{l}\text { Weit off } \\
\text { velue }\end{array}$ & Metrics & PREDICT & PD-MRW & $\begin{array}{l}\text { PDO- } \\
\text { MSSARW }\end{array}$ & $\begin{array}{l}\text { PDO- } \\
\text { MSBARW }\end{array}$ & $\begin{array}{l}\text { PDDH } \\
\text { MICARM }\end{array}$ & $\begin{array}{l}\text { PDO- } \\
\text { MCSRWA }\end{array}$ \\
\hline \multirow[t]{4}{*}{$w=5$} & Precision (\$6) & 89.76 & 90.02 & 9105 & 92.56 & 96.98 & 97.35 \\
\hline & Reall (36) & 88.70 & 89.91 & 90.10 & 91.34 & 96.98 & 97.48 \\
\hline & F-messure $[3 /$ & 89.23 & 89.96 & 90.57 & 91.95 & 96.98 & 97.53 \\
\hline & Accurecy $[3 \%$ & 86.35 & 89.71 & 91.82 & 92.76 & 94.40 & 96.21 \\
\hline \multirow[t]{4}{*}{$w=10$} & Precision (3/6) & 91.18 & 93.38 & 95.56 & 96.45 & 96.96 & 97.59 \\
\hline & Reall (\%) & 91.11 & 92.31 & 98.48 & 94.32 & 97.81 & 98.32 \\
\hline & F-messure $[/ 6]$ & 91.14 & 92.84 & 94.52 & 95.38 & 97.38 & 98.21 \\
\hline & Accurscy $[\%]$ & 91.25 & 92.49 & 98.53 & 94.65 & 95.20 & 97.23 \\
\hline \multirow[t]{4}{*}{$w=15$} & Precision $(36)$ & 92.60 & 94.22 & 96.77 & 97.12 & 97.83 & 98.21 \\
\hline & Reall (\%) & 94.57 & 95.19 & 96.77 & 97.21 & 97.83 & 98.36 \\
\hline & F-messure $(\beta / 6)$ & 93.58 & 94.70 & 96.77 & 97.16 & 97.83 & 98.11 \\
\hline & Accurecy $[\%]$ & 92.64 & 93.41 & 94.75 & 95.68 & 96.00 & 97.52 \\
\hline
\end{tabular}
medication classifications.

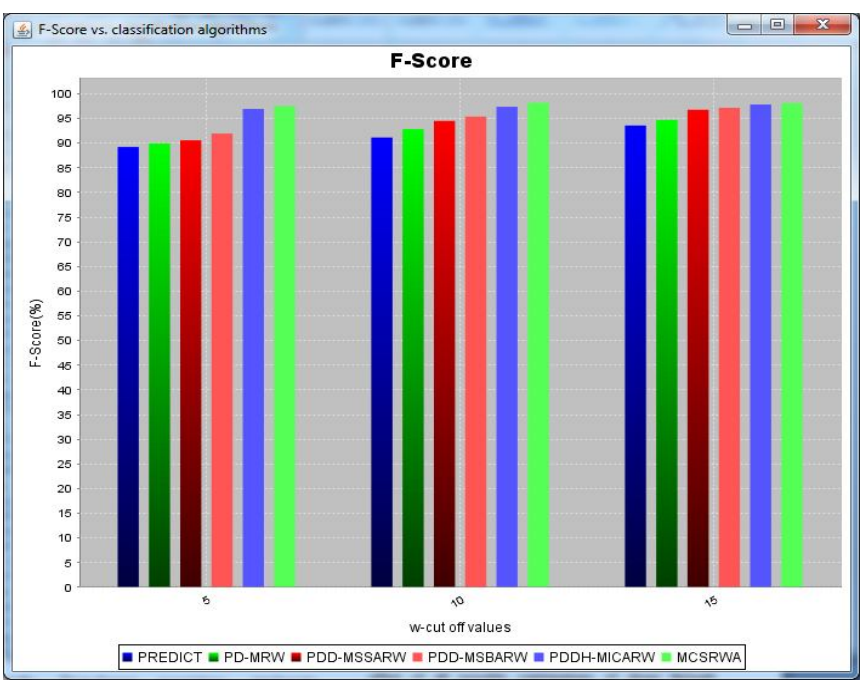

Figure 2: F-measure vs. methods (with different weight values)

In Figure 2, the outcomes of the f-measure interpretation of the three different weight $w$ value cutoffs have shown among 
six PDD-MCSRWA methods PDDH-MICARW, PDD-MSBARW, PDD-MSSARW, PD-MRW, and PREDICT. The prospective PDD-MCSRWA approach gives the high caliber f-measurement of 98,11 percent for $\mathrm{w}=15$ when the F-measurement of 97, 83 percent, 97,165 percent, and 94,705 percent for 97, 15 percent for other approaches such as PDDH-MICARW, PDD-MSBARW, PDD-MSSARW PD-MRW.

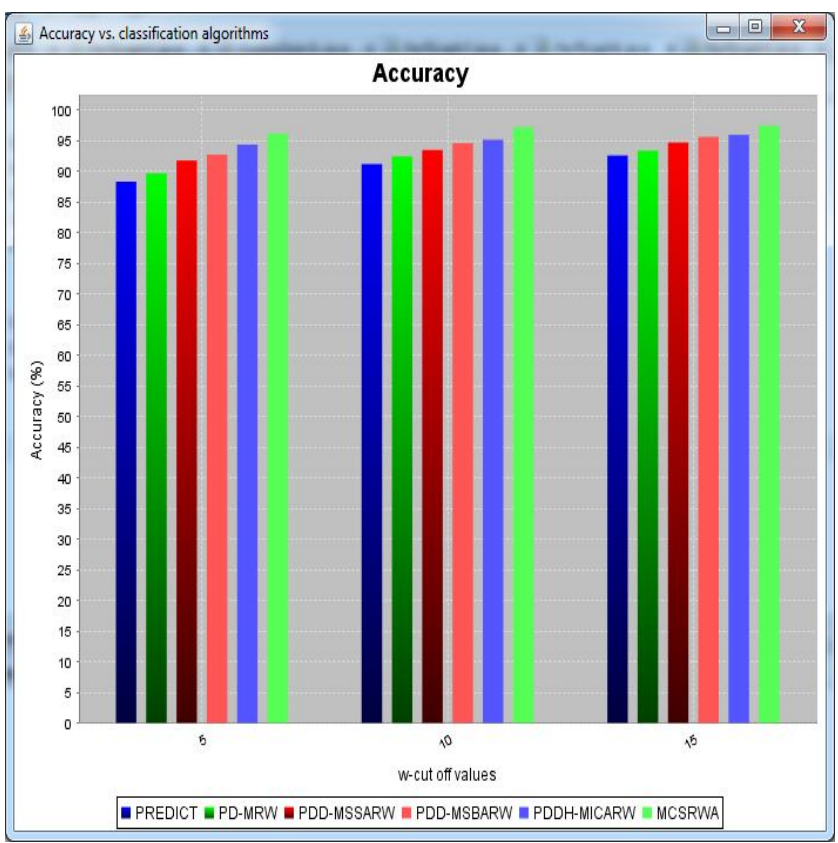

Figure 3:: Accuracy vs. methods (with different weight values)

In Figure 3, the suggested PDD-MCSRWA method presents an increase in the precision rate of $97.52 \%$ for $\mathrm{w}=15$ with the provision of precision by other methods, such as the PDDH-MICARW, the PDD-MSBARW, the PDD-MSSARW, the PD-MRW, and PREDICT technology of $96.00 \%, 95.68 \%$, $94.41 \%$, and $92.64 \%$, respectively.

\section{CONCLUSION AND FUTURE WORK}

The repositioning of drugs is one of the most important methods of improving drug discovery processes. Many current drug-repositioning methods are not focused on a specific disease and are likely to use a specific information source to define interactions between pharmaco-disease.

In this original paper, the focus is on Hepatocellular carcinoma (HCC) as well as new Predict New Drugs as well as HCC-dependent Diseases, based on the Random Walk (PDD-MSSARW) multi-source simulation method[ 18] for the forecast of fresh use of medicines.

The second job, a multiple-source bat algorithm oriented Random Walk (PDD-MBARW)[ 19] is used as an inclusive structure. A new improved SVD algorithm for a decrease of dimensionality will provided which will increase the accuracy of the forecast. Finally, the network of Medicine-Similarity, the network of bipartite-based disease-drugs has based on the recognized HCC drugs. This network provides further information regarding these relationships, combined with the parent or element networks.

Improved Linear Algorithm of Regularized Analysis for Discrimination (IRLDA), third input, is being suggested to reducing dimensionality. The suggested method PDDH-MICARW integrates diverse data and knowledge to the random cross-source hypothesis a resemblance of each drug couple.

The third entry, enhanced and It is suggested that the decrease in dimension should be lowered by regularized linear discrimination analyzes (IRLDA). The PDDH-MICARW technology suggested the random cross-source hypothesis integrates heterogeneous information and expertise and makes use of it to achieve a resemblance among individual compounds.

Finally, all medicines have recorded in the drug resemblance network depending on the recognized CCA medicines. The experimental results show that the suggested technique obtained higher results in precision, prediction, reclamation and F-measurement in line with current methods. The assessment of the accuracy of the statistical solution involves a certain matter whereby the data related to the dimension curse, the data has not monitored, or the supervising information that remains within the scope of future work is insufficient. In future job is concentrated on the application of the suggested technique by taking into account signs of disease and side effects of medicines.

\section{REFERENCES}

1. Wilson JF. Alterations in processes and priorities needed for new drug development. Ann Intern Med. 2006; 145(10):793-796.

https://doi.org/10.7326/0003-4819-145-10-200611210-00024

2. Ferlay, J., Steliarova-Foucher, E., Lortet-Tieulent, J., Rosso, S., Coebergh, J. W. W., Comber, H., \& Bray, F.. Cancer incidence and mortality patterns in Europe: estimates for 40 countries in 2012. European journal of cancer, 49(6), 1374-1403(2013). https://doi.org/10.1016/j.ejca.2012.12.027

3. Wu, J. (2017). The changing epidemiology of hepatocellular carcinoma in Asia versus the United States and Europe. Advances in Modern Oncology Research, 3(s1), 51-58. https://doi.org/10.18282/amor.v3.is1.182

4. Gottlieb, A., Stein, G.Y., Ruppin, E., and Sharan, R., 2011. PREDICT a method for inferring novel drug 
indications with application to personalized medicine. Molecular systems biology, 7(1), pp.1-15.

https://doi.org/10.1038/msb.2011.26

5. Li J, Zhu X, Chen J. Building disease-specific drug-protein connectivity maps from molecular interaction networks and PubMed abstracts. PLoS Comput Biol. 2009; 5(7) : e1000450. https://doi.org/10.1371/journal.pcbi.1000450

6. Yu, L., Xu, F., \& Gao, L. (2017). Predicting therapeutic and aggravating drugs for hepatocellular carcinoma based on tissue-specific pathways. arXiv preprint arXiv:1707.00954.

7. Luo, Y., Zhao, X., Zhou, J., Yang, J., Zhang, Y., Kuang, W., ... \& Zeng, J. (2017). A network integration approach for drug-target interaction prediction and computational drug repositioning from heterogeneous information. Nature Communications, 8(1), 573. https://doi.org/10.1038/s41467-017-00680-8

8. Alan M. Frieze, Colin Cooper, SIAM J, Tomasz Radzik, "Multiple Random Walks in Random Regular Graphs," Discrete Math, 23, pp. 1738-1761, 2009. https://doi.org/10.1137/080729542

9. Aarts, E., Korst, J. and Michiels, W., 2005. Simulated annealing. In Search methodologies (pp. 187-210). Springer, Boston, MA. https://doi.org/10.1007/0-387-28356-0_7

10. Li, Z., Wu, X.M. and Chang, S.F., 2012, Segmentation using superpixels: A bipartite graph partitioning approach. IEEE Conference on Computer Vision and Pattern Recognition (CVPR), pp. 789-796.

11. Alan M. Frieze, Colin Cooper, SIAM J, Tomasz Radzik, "Multiple Random Walks in Random Regular Graphs," Discrete Math, 23, pp. 1738-1761, 2009. https://doi.org/10.1137/080729542

12. X.-S. Yang: A New Metaheuristic Bat-Inspired Algorithm, in Nature Inspired Cooperative Strategies for Optimization (NICSO 2010), Studies in Computational Intelligence 284, Edited J. R. González, D. A. Pelta, C. Cruz, G. Terrazas, and N. Krasnogor, Springer-Verlag, Berlin Heidelberg (2010), pp. 65-74.

13. Atashpaz-Gargari, E.; Lucas, C. Imperialist competitive algorithm for minimum bit error rate beamforming. Int. J. Bio-Inspired Comput. 2009, 1, pp.125-133.

https://doi.org/10.1504/IJBIC.2009.022781

14. Davis, A.P., Grondin, C.J., Lennon-Hopkins, K., Saraceni-Richards, C., Sciaky, D., King, B.L., Wiegers, T.C. and Mattingly, C.J., 2014. The Comparative Toxicogenomics Database's 10th year anniversary: update 2015. Nucleic acids research, 43(D1), pp.D914-D920.4

https://doi.org/10.1093/nar/gku935

15. Barrett, T., Wilhite, S.E., Ledoux, P., Evangelista, C., Kim, I.F., Tomashevsky, M., Marshall, K.A., Phillippy, K.H., Sherman, P.M., Holko, M. and Yefanov, A., 2012. NCBI GEO: archive for functional genomics data sets-update. Nucleic acids research, 41(D1), pp.D991-D995.

https://doi.org/10.1093/nar/gks1193

16. Wishart, D.S., Knox, C., Guo, A.C., Shrivastava, S., Hassanali, M., Stothard, P., Chang, Z. and Woolsey, J., 2006. DrugBank: a comprehensive resource for in silico drug discovery and exploration. Nucleic acids research, 34(suppl_1), pp.D668-D672. https://doi.org/10.1093/nar/gkj067

17. Law, V., Knox, C., Djoumbou, Y., Jewison, T., Guo, A.C., Liu, Y., Maciejewski, A., Arndt, D., Wilson, M., Neveu, V. and Tang, A., 2013. DrugBank 4.0: shedding new light on drug metabolism. Nucleic acids research, 42(D1), pp.D1091-D1097. https://doi.org/10.1093/nar/gkt1068

18. Ibrahim, S. Jafar Ali, and M. Thangamani. "Prediction of Novel Drugs and Diseases for Hepatocellular Carcinoma Based on Multi-Source Simulated Annealing Based Random Walk." Journal of medical systems 42, no. 10 (2018): 188. https://doi.org/10.1007/s10916-018-1038-y

19. Ibrahim, S. Jafar Ali, and M. Thangamani. "Enhanced singular value decomposition for prediction of drugs and diseases with Hepatocellular carcinoma based on Multi-Source Bat Algorithm based random walk." Measurement 141 (2019): 176-183. https://doi.org/10.1016/j.measurement.2019.02.056

20. Nouf Saeed Alotaibi, "An Efficient Technique for Reducing Transmission Energy of Sensor Node to Enhance the Lifetime of the WSN's" International Journal of Advanced Trends in Computer Science and Engineering Volume 8, No.2, PP.108-111. https://doi.org/10.30534/ijatcse/2019/02822019

21. Maria Zemzami, Aicha Koulou, Norelislam Elhami, Mhamed Itmi, Nabil Hmina "Interoperability Optimization using a modified PSO algorithm" International Journal of Advanced Trends in Computer Science and Engineering Volume 8, No.2, PP.101-107. https://doi.org/10.30534/ijatcse/2019/01822019 